\title{
CHARACTERIZATIONS OF CLASSES OF HARMONIC CONVEX FUNCTIONS AND APPLICATIONS
}

\section{IMRAN ABBAS BALOCH ${ }^{1,2, *}$, MANUEL DE LA SEN ${ }^{3}$ AND İMDAT İSCAN ${ }^{4}$}

\author{
${ }^{1}$ Abdus Salam School of Mathematical Sciences, GC University, Lahore, Pakistan \\ Govt. College for Boys Gulberg, Higher Education Department, Punjab, Pakistan \\ ${ }^{3}$ Institute of Research and Development of Processors, University of the Basque, Country Campus of Leioa \\ (Bizkaia), 48940 Leioa, Spain \\ ${ }^{4}$ Department of Mathematics, Faculty of Arts and Sciences, Giresun University, 28100, Giresun, Turkey
}

*Corresponding author: iabbasbaloch@gmail.com, iabbasbaloch@sms.edu.pk

\begin{abstract}
In this paper, we consider classes of harmonic convex functions and give their special characterizations. Furthermore, we consider Hermite Hadamard type inequalities related to these classes to give some non-numeric estimates of well-known definite integrals.
\end{abstract}

\section{Introduction}

The classical or the usual convexity is defined as follows:

A function $f: \emptyset \neq I \subseteq \mathbb{R} \longrightarrow \mathbb{R}$, is said to be convex on $I$ if inequality

$$
f(t x+(1-t) y) \leq t f(x)+(1-t) f(y)
$$

holds for all $x, y \in I$ and $t \in[0,1]$.

Received 2019-06-23; accepted 2019-08-01; published 2019-09-02.

2010 Mathematics Subject Classification. 26D15, 26A51, 26D10, 26A15.

Key words and phrases. harmonic convex functions; harmonic $(\mathrm{p},(\mathrm{s}, \mathrm{m}))$-convex functions; Hermite Hadamard inequalities; well-known definite integrals.

(C)2019 Authors retain the copyrights of their papers, and all open access articles are distributed under the terms of the Creative Commons Attribution License. 
A number of papers have been written on inequalities using the classical convexity and one of the most fascinating inequalities in mathematical analysis is stated as follows

$$
f\left(\frac{a+b}{2}\right) \leq \frac{1}{b-a} \int_{a}^{b} f(x) d x \leq \frac{f(a)+f(b)}{2},
$$

where $f: I \subseteq \mathbb{R} \longrightarrow \mathbb{R}$ be a convex mapping and $a, b \in I$ with $a \leq b$. Both the inequalities hold in reversed direction if $f$ is concave. The inequalities stated in (1.1) are known as Hermite-Hadamard inequalities.

For more results on (1.1) which provide new proof, significantly extensions, generalizations, refinements, counterparts, new Hermite-Hadamard-type inequalities and numerous applications, we refer the interested reader $[1-4,14]$ and the references therein.

The usual notion of convex function have been generalized in diverse manners. Some of them are the so called harmonically convex function, harmonically $(\alpha, m)$-convex function and $p$-convex function, which are stated in the definitions below.

Definition 1.1. [9] A function $f: I \subseteq \mathbb{R} \backslash\{0\} \rightarrow \mathbb{R}$ is said to be harmonically-convex function on $I$ if

$$
f\left(\frac{x y}{t x+(1-t) y}\right) \leq t f(y)+(1-t) f(x)
$$

holds for all $x, y \in I$ and $t \in[0,1]$. If the inequality is reversed, then $f$ is said to be harmonically concave.

Definition 1.2. [10] A function $f: I \subseteq \mathbb{R} \backslash\{0\} \rightarrow \mathbb{R}$ is said to be harmonically $(\alpha, m)$-convex function on I if

$$
f\left(\frac{m x y}{m t y+(1-t) x}\right) \leq t^{\alpha} f(x)+m\left(1-t^{\alpha}\right) f(y)
$$

holds for all $x, y \in I$ and $t \in[0,1]$, where $\alpha \in[0,1]$ and $m \in(0,1]$. If the inequality is reversed, then $f$ is said to be harmonically $(\alpha, m)$-concave.

Definition 1.3. [11,13] Let $I \subseteq(0, \infty)$ be a real interval. A function $f: I \rightarrow \mathbb{R}$ is p-convex, where $p \in \mathbb{R} /\{0\}$ if

$$
f\left(\left[t x^{p}+(1-t) y^{p}\right]^{\frac{1}{p}}\right) \leq t f(y)+(1-t) f(x)
$$

holds for all $x, y \in I$ and $t \in[0,1]$. If the inequality is reversed, then $f$ is said to be $p$-concave.

\section{Main Results}

The convexity of functions and their generalized forms, play an important role in many fields such as Economic Science, Biology, Optimization. In recent years, several extensions, refinements, and generalizations have been considered for classical convexity. In [7], I. A. Baloch and $\dot{I}$. $\dot{I}_{\text {şcan defined a new class of }}$ functions which is defined as follow: 
Definition 2.1. [6, 7] A function $f: I \subseteq \mathbb{R} \backslash\{0\} \rightarrow \mathbb{R}$ is said to be harmonically $(s, m)$-convex function on I if

$$
f\left(\frac{m x y}{m t y+(1-t) x}\right) \leq t^{s} f(x)+m(1-t)^{s} f(y)
$$

holds for all $x, y \in I$ and $t \in[0,1]$, where $s \in(0,1]$ and $m \in(0,1]$. If the inequality is reversed, then $f$ is said to be harmonically $(s, m)$-concave.

Definition 2.2. [5] A function $f: I \subseteq \mathbb{R} \backslash\{0\} \rightarrow \mathbb{R}$ is said to be harmonically $(p,(s, m))$-convex function, where $p \in \mathbb{R} /\{0\}, s, m \in(0,1]$, if

$$
f\left(\frac{m x y}{\left[t(m y)^{p}+(1-t) x^{p}\right]^{\frac{1}{p}}}\right) \leq t^{s} f(x)+m(1-t)^{s} f(y),
$$

for all $x, y \in I$ with $m y \in I$ and $t \in[0,1]$. If the inequality is reversed, then $f$ is said to be harmonically $(p,(s, m))$-concave.

Now, first of all, we give an example of harmonic convex function which is not a convex function.

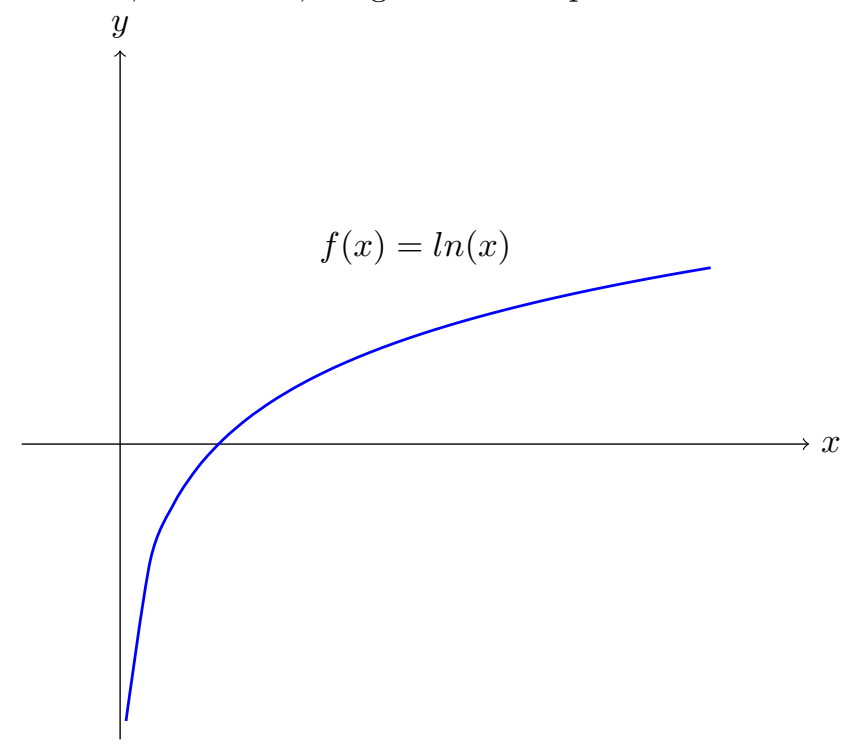

Here, some more examples of harmonic convex functions.

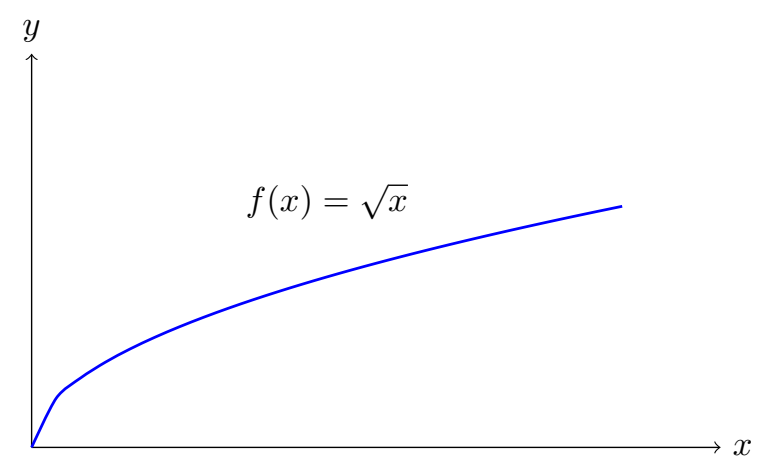



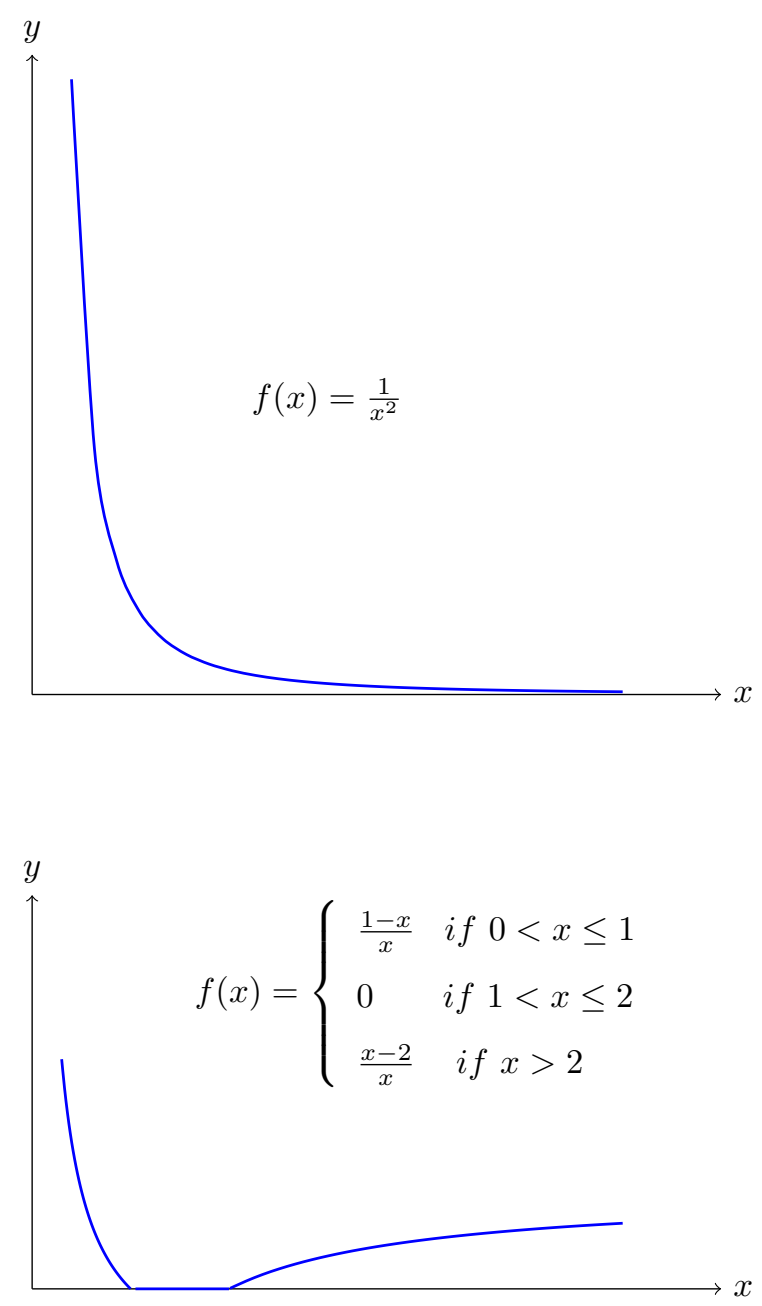

Lemma 2.1. Let $I \subseteq \mathbb{R} /\{0\}$ be a real interval. Define $I^{-1}=\left\{y \in \overline{\mathbb{R}}, y=\frac{1}{x}, x \in I\right\}$.

A function $f: I \rightarrow \mathbb{R}$ is harmonically convex if and only if $g: I^{-1} \rightarrow \mathbb{R}$ is convex, where $g$ is defined as $g(y)=f\left(\frac{1}{y}\right)$.

Proof. Let $f$ be harmonically convex function on $I$ and consider a function $g: I^{-1} \rightarrow \mathbb{R}$ defined as $g(y)=$ $f\left(\frac{1}{y}\right)$. Now, $\forall y_{1}, y_{2} \in I^{-1}, \exists x_{1}, x_{2} \in I$ such that $y_{1}=\frac{1}{x_{1}}$ and $y_{2}=\frac{1}{x_{2}}$

$$
\begin{aligned}
g\left(t y_{1}+(1-t) y_{2}\right) & =f\left(\frac{1}{t y_{1}+(1-t) y_{2}}\right) \\
& =f\left(\frac{1}{t \frac{1}{x_{1}}+(1-t) \frac{1}{x_{2}}}\right) \\
& =f\left(\frac{x_{1} x_{2}}{t x_{2}+(1-t) x_{1}}\right) \\
& \leq t f\left(x_{1}\right)+(1-t) f\left(x_{2}\right) \\
& =t f\left(\frac{1}{y_{1}}\right)+(1-t) f\left(\frac{1}{y_{1}}\right) \\
& +t g\left(y_{1}\right)+(1-t) g\left(y_{2}\right) .
\end{aligned}
$$


From above, we conclude the proof.

Lemma 2.2. [12] Let $f$ be a real function defined on an interval $I \subseteq \mathbb{R}$. Then, $f$ is convex if and only if

$$
\left|\begin{array}{lll}
1 & x & f(x) \\
1 & y & f(y) \\
1 & z & f(z)
\end{array}\right| /\left|\begin{array}{lll}
1 & x & x^{2} \\
1 & y & y^{2} \\
1 & z & z^{2}
\end{array}\right| \geq 0
$$

for all three distinct points $x, y, z$ of $I$; equivalently, if and only if

$$
\left|\begin{array}{lll}
1 & x & f(x) \\
1 & y & f(y) \\
1 & z & f(z)
\end{array}\right| \geq 0
$$

for all $x<y<z$ in $I$.

Lemma 2.3. Let $I \subseteq(0, \infty)$ and $I^{-1}$ has a similar definition as given in Lemma 2.1. A function $f: I \rightarrow \mathbb{R}$ is harmonically convex if and only if $h: I \rightarrow \mathbb{R}$ is convex, where $h$ is defined as $h(z)=z f(z)$.

Proof. From the Lemma 2.1, a function $f$ is harmonically convex function on $I$ if and only if $g(y)=f\left(\frac{1}{y}\right)$ is convex on $I^{-1}$ and by Lemma 2.2 , a function $g(y)$ is convex if and only if

$$
\left|\begin{array}{lll}
1 & y_{1} & g\left(y_{1}\right) \\
1 & y_{2} & g\left(y_{2}\right) \\
1 & y_{3} & g\left(y_{3}\right)
\end{array}\right| \geq 0
$$

for all $y_{1}, y_{2}, y_{3} \in I^{-1}$ such that $y_{1}<y_{2}<y_{3}$. Equivalently

$$
\left|\begin{array}{lll}
1 & y_{1} & f\left(\frac{1}{y_{1}}\right) \\
1 & y_{2} & f\left(\frac{1}{y_{2}}\right) \\
1 & y_{3} & f\left(\frac{1}{y_{3}}\right)
\end{array}\right| \geq 0
$$

for all $y_{1}, y_{2}, y_{3} \in I^{-1}$ such that $y_{1}<y_{2}<y_{3}$.

Equivalently

$$
\left|\begin{array}{lll}
x_{1} & 1 & x_{1} f\left(x_{1}\right) \\
x_{2} & 1 & x_{2} f\left(x_{2}\right) \\
x_{3} & 1 & x_{3} f\left(x_{3}\right)
\end{array}\right| \geq 0
$$


for all $x_{1}, x_{2}, x_{3} \in I$ such that $x_{1}>x_{2}>x_{3}$. Now, by interchanging the first and third row, first and second column, and relabeling $x_{3}=z_{1}, x_{2}=z_{2}$ and $x_{1}=z_{3}$, we arrive at

$$
\left|\begin{array}{ccc}
1 & z_{1} & z_{1} f\left(z_{1}\right) \\
1 & z_{2} & z_{2} f\left(z_{2}\right) \\
1 & z_{3} & z_{3} f\left(z_{3}\right)
\end{array}\right| \geq 0
$$

for all $z_{1}, z_{2}, z_{3} \in I$ such that $z_{1}<z_{2}<z_{3}$, which is equivalent to the convexity of the function $h(z)=z f(z)$ on $I$.

Proposition 2.1. [9] Let $I \subseteq \mathbb{R} /\{0\}$ be a real interval and $f: I \rightarrow \mathbb{R}$ is a function, then ;

1) If $I \subset(0, \infty)$ and $f$ is convex and nondecreasing function, then $f$ is a harmonically convex function.

2) If $I \subset(0, \infty)$ and $f$ is a harmonically convex and nonincreasing function, then $f$ is convex function.

3) If $I \subset(-\infty, 0)$ and $f$ is a harmonically convex and nondecreasing function, then $f$ is convex function.

4) If $I \subset(-\infty, 0)$ and $f$ is convex and nonincreasing function, then $f$ is a harmonically convex function.

Proof. The proof is immediate by following inequality

$$
0 \leq t(1-t)(x-y)^{2}
$$

for all $x, y \in I$. Equivalently,

$$
\frac{x y}{t x+(1-t) y} \leq t y+(1-t) x
$$

for all $x, y \in(0, \infty)$, and

$$
\frac{x y}{t x+(1-t) y} \geq t y+(1-t) x
$$

for all $x, y \in(-\infty, 0)$.

\section{Examples:}

- Let $f:(0, \infty) \rightarrow \mathbb{R}, f(x)=x$ and $g:(-\infty, 0) \rightarrow \mathbb{R}, g(x)=x$, then $f$ is a harmonically convex function and $g$ is a harmonically concave function.

- Let $f:(0, \infty) \rightarrow \mathbb{R}$,

$$
f(x)= \begin{cases}x, & 0<x<2 \\ 4-\frac{4}{x}, & x \geq 2\end{cases}
$$

is harmonically convex function on $(0, \infty)$, since $x f(x)$ is convex on $(0, \infty)$.

- Let $f:(0, \infty) \rightarrow \mathbb{R}$,

$$
f(x)=\frac{(x-1)^{2}+1}{x}
$$

is a harmonically convex function on $(0, \infty)$, since $x f(x)$ is convex on $(0, \infty)$. 
- By the Proposition 2.1, increasing convex function is harmonically convex. This means that $e^{x}$ is harmonically convex.

The following result of the Hermite-Hadamard type holds for class of harmonically convex functions.

Theorem 2.1. [9] Let $f: I \subseteq \mathbb{R} /\{0\} \rightarrow \mathbb{R}$ be harmonically convex and $a, b \in I$ with $a<b$. If $f \in L[a, b]$, then following inequalities hold

$$
f\left(\frac{2 a b}{a+b}\right) \leq \frac{(a b)}{b-a} \int_{a}^{b} \frac{f(x)}{x^{2}} d x \leq \frac{f(a)+f(b)}{2}
$$

The above inequalities are sharp.

Remark 2.1. Since, $f(x)=\ln x$ is harmonic convex on $(0, \infty)$, therefore by inequality (2.2) we have

$$
\frac{2 a b}{a+b} \leq e\left[\frac{a^{b}}{b^{a}}\right]^{\frac{1}{b-a}} \leq \sqrt{a b}
$$

Now, using Lemma 2.3, we get following interesting result.

Theorem 2.2. Let $I \subseteq(0, \infty)$ be a real interval. If function $f: I \rightarrow \mathbb{R}$ is harmonically convex and a, $b \in I$ with $a<b$ such that $f \in L[a, b]$, then the following inequalities hold

$$
\left(\frac{a+b}{2}\right) f\left(\frac{a+b}{2}\right) \leq \frac{1}{b-a} \int_{a}^{b} x f(x) d x \leq \frac{a f(a)+b f(b)}{2}
$$

Proof. Since, $f(x)$ is harmonically convex on $I$, therefore by Lemma $2.3 x f(x)$ is convex on $I$. Hence, by the use of inequality (1.1), we get required result.

Remark 2.2. Since, $f(x)=\ln x$ is harmonic convex on $(0, \infty)$, therefore by inequality (2.3) we have

$$
\left(\frac{a+b}{2}\right)^{\frac{a+b}{2}} \leq e^{-\frac{a+b}{2}} \cdot\left[\frac{b^{b^{2}}}{a^{a^{2}}}\right]^{\frac{1}{b-a}} \leq \sqrt{a^{a} b^{b}}
$$

Theorem 2.3. [8] Let $I \subseteq \mathbb{R}$ be a real interval. If function $f: I \rightarrow \mathbb{R}$ is $p$-convex, where $p \in \mathbb{R} /\{0\}$ and $a, b \in I$ with $a<b$ such that $f \in L[a, b]$, then following inequalities hold

$$
f\left(\left[\frac{a^{p}+b^{p}}{2}\right]^{\frac{1}{p}}\right) \leq \frac{p}{b^{p}-a^{p}} \int_{a}^{b} \frac{f(x)}{x^{1-p}} d x \leq \frac{f(a)+f(b)}{2}
$$

Lemma 2.4. Let $I \subseteq \mathbb{R} /\{0\}$ be a real interval and $I^{-1}$ has similar definition as given in Lemma 2.1. A function $f: I \rightarrow \mathbb{R}$ is harmonically $p$-convex if and only if $g(y)=f\left(\frac{1}{y}\right)$ is p-convex. 
Proof. Let $f$ be a function on $I$ and consider a function $g: I^{-1} \rightarrow \mathbb{R}$ defined as $g(y)=f\left(\frac{1}{y}\right)$, is $p$-convex function. Now, $\forall y_{1}, y_{2} \in I^{-1}, \exists x_{1}, x_{2} \in I$ such that $y_{1}=\frac{1}{x_{1}}$ and $y_{2}=\frac{1}{x_{2}}$

$$
\begin{aligned}
f\left(\frac{x_{1} x_{2}}{\left[t x_{2}^{p}+(1-t) x_{1}^{p}\right]^{\frac{1}{p}}}\right) & =f\left(\frac{1}{\left[t y_{1}^{p}+(1-t) y_{2}^{p}\right]^{\frac{1}{p}}}\right) \\
& =g\left(\left[t y_{1}^{p}+(1-t) y_{2}^{p}\right]^{\frac{1}{p}}\right) \\
& \leq t g\left(y_{1}\right)+(1-t) g\left(y_{2}\right) \\
& =t f\left(\frac{1}{y_{1}}\right)+(1-t) f\left(\frac{1}{y_{2}}\right) \\
& =t f\left(x_{1}\right)+(1-t) f\left(x_{2}\right)
\end{aligned}
$$

From above, we conclude the proof.

Lemma 2.5. Let $I \subseteq \mathbb{R}$ be a real interval. A function $f: I \rightarrow \mathbb{R}$ is a p-convex function if and only if

$$
\left|\begin{array}{lll}
1 & x^{p} & f(x) \\
1 & y^{p} & f(y) \\
1 & z^{p} & f(z)
\end{array}\right| \geq 0
$$

for all $x, y, z \in I$ such that $x<y<z$.

Lemma 2.6. Let $I \subseteq(0, \infty)$ be a real interval and $I^{-1}$ has similar definition as given in Lemma 2.1. A function $f: I \rightarrow \mathbb{R}$ is harmonically p-convex if and only if $h: I \rightarrow \mathbb{R}$ defined by $h(z)=z^{p} f(z)$ is convex.

Proof. From the Lemma 2.4, a function $f$ is a harmonically $p$-convex function on $I$ if and only if $g(y)=f\left(\frac{1}{y}\right)$ is $p$-convex and by Lemma 2.5 , a function $g(x)$ is $p$-convex if and only if

$$
\left|\begin{array}{lll}
1 & y_{1}^{p} & g\left(y_{1}\right) \\
1 & y_{2}^{p} & g\left(y_{2}\right) \\
1 & y_{3}^{p} & g\left(y_{3}\right)
\end{array}\right| \geq 0
$$

for all $y_{1}, y_{2}, y_{3} \in I$ such that $y_{1}<y_{2}<y_{3}$. Equivalently

$$
\left|\begin{array}{ccc}
1 & \frac{1}{x_{1}^{p}} & f\left(x_{1}\right) \\
1 & \frac{1}{x_{2}^{p}} & f\left(x_{2}\right) \\
1 & \frac{1}{x_{3}^{p}} & f\left(x_{3}\right)
\end{array}\right| \geq 0
$$

for all $x_{1}, x_{1}, x_{1} \in I$ such that $x_{1}>x_{2}>x_{3}$. Now, by the multiplying the ith row of the determinant by $x_{i}^{p}$, interchanging the first and third row, first and second column, and relabeling $x_{3}=z_{1}, x_{2}=z_{2}$ and $x_{1}=z_{3}$, we arrive at 


$$
\left|\begin{array}{rrr}
1 & z_{1}^{p} & z_{1}^{p} f\left(z_{1}\right) \\
1 & z_{2}^{p} & z_{2}^{p} f\left(z_{2}\right) \\
1 & z_{3}^{p} & z_{3}^{p} f\left(z_{3}\right)
\end{array}\right| \geq 0
$$

for all $z_{1}, z_{2}, z_{3} \in I$ such that $z_{1}<z_{2}<z_{3}$, which is equivalent to the convexity of the function $h(z)=$ $z^{p} f(z)$

Proposition 2.2. Let $I \subseteq \mathbb{R} /\{0\}$ be a real interval, $p \in \mathbb{R} /\{0\}$ and $f: I \rightarrow \mathbb{R}$ is a function, then;

- If $p \geq 1$ and harmonically convex and nondecreasing function, then $f$ is harmonically $p$-convex function.

- If $p \geq 1$ and harmonically p-convex and nonincreasing function, then $f$ is harmonically convex function.

- If $p \geq 1$ and harmonically $p$-concave and nondecreasing function, then $f$ is harmonically concave function.

- If $p \geq 1$ and harmonically concave and nonincreasing function, then $f$ is harmonically p-concave function.

- If $p \leq 1$ and harmonically p-convex and nondecreasing function, then $f$ is harmonically convex function.

- If $p \leq 1$ and harmonically convex and nonincreasing function, then $f$ is harmonically $p$-convex function.

- If $p \leq 1$ and harmonically concave and nondecreasing function, then $f$ is harmonically p-concave function.

- If $p \leq 1$ and harmonically p-concave and nonincreasing function, then $f$ is harmonically concave function.

Proof. Since $f(x)=x^{p}, p \in(-\infty, 0) \cup[1, \infty)$, is convex function on $(0, \infty)$ and $f(x)=x^{p}, p \in(0,1]$, is a concave function on $(0, \infty)$, therefore the proof is obvious from the following power mean inequalities

$$
\left[t x^{p}+(1-t) y^{p}\right]^{\frac{1}{p}} \geq t x+(1-t) y, \quad p \geq 1
$$

which is equivalent to

$$
\frac{x y}{\left[t x^{p}+(1-t) y^{p}\right]^{\frac{1}{p}}} \leq \frac{x y}{t x+(1-t) y}, \quad p \geq 1
$$


and

$$
\left[t x^{p}+(1-t) y^{p}\right]^{\frac{1}{p}} \leq t x+(1-t) y, \quad p \leq 1
$$

which is equivalent to

$$
\frac{x y}{t x+(1-t) y} \leq \frac{x y}{\left[t x^{p}+(1-t) y^{p}\right]^{\frac{1}{p}}}, \quad p \geq 1,
$$

for all $x, y \in(0, \infty)$ and $t \in[0,1]$.

Similar to inequality given in Theorem 2.1, we proved the result for harmonically $p$-convex functions as given below

Theorem 2.4. [5] Let $I \subseteq \mathbb{R} /\{0\}$ be a real interval and $f: I \rightarrow \mathbb{R}$ be harmonically $p$-convex, $a, b \in I$ with $a<b$. If $f \in L[a, b]$, then following inequalities hold

$$
f\left(\frac{2^{\frac{1}{p}} a b}{\left[a^{p}+b^{p}\right]^{\frac{1}{p}}}\right) \leq \frac{p(a b)^{p}}{b^{p}-a^{p}} \int_{a}^{b} \frac{f(x)}{x^{p+1}} d x \leq \frac{f(a)+f(b)}{2} .
$$

Also, by using Lemma 2.6 and Theorem 3, we have another interesting result as

Theorem 2.5. Let $I \subseteq(0, \infty)$ be a real interval and $I^{-1}$ has similar definition as given in Lemma 2.1. If function $f: I \rightarrow \mathbb{R}$ is harmonically $p$-convex, where $p \in \mathbb{R} /\{0\}$ and $a, b \in I$ with $a<b$ such that $f \in L[a, b]$, then following inequalities hold

$$
\left(\frac{a^{p}+b^{p}}{2}\right) f\left(\left[\frac{a^{p}+b^{p}}{2}\right]^{\frac{1}{p}}\right) \leq \frac{p}{b^{p}-a^{p}} \int_{a}^{b} \frac{f(x)}{x^{1-2 p}} d x \leq \frac{a^{p} f(a)+b^{p} f(b)}{2} .
$$

\section{Applications}

- we have relation between Harmonic, Logarithmic and Arithmetic means as follow

$$
\frac{2 a b}{a+b} \leq \frac{a b}{b-a}(\ln b-\ln a) \leq \frac{a+b}{2}(\text { HLA inequality })
$$

whose proof is a direct consequence of the inequality of Theorem 2.1 for $f(x)=x, \forall x \in(0, \infty)$.

Furthermore, we have following consequence of the inequality of Theorem 2 for $f(x)=x, \forall x \in(0, \infty)$.

$$
\left(\frac{a+b}{2}\right)^{2} \leq \frac{1}{3}\left(a^{2}+a b+b^{2}\right) \leq \frac{a^{2}+b^{2}}{2} .
$$

Even in more general setting for $p \in \mathbb{R} /\{0,1\}$, we have

$$
\frac{2^{\frac{1}{p}} a b}{\left[a^{p}+b^{p}\right]^{\frac{1}{p}}} \leq \frac{p(a b)^{p}}{b^{p}-a^{p}}\left(\frac{b^{1-p}-a^{1-p}}{1-p}\right) \leq \frac{a+b}{2}
$$


which is a direct consequence of inequality of Theorem 2.4 and we have

$$
\left(\frac{a^{p}+b^{p}}{2}\right)^{\frac{p+1}{p}} \leq \frac{p}{b^{p}-a^{p}} \cdot \frac{b^{2 p+1}-a^{2 p-1}}{2 p+1} \leq \frac{a^{p+1}+b^{p+1}}{2} .
$$

which is a direct consequence of inequality of Theorem 5 for $f(x)=x, \forall x \in(0, \infty)$.

- For $a, b \in(0, \infty)$ and $a \neq b$, we have

$$
e^{\frac{2 a b}{a+b}} \leq \frac{a b}{b-a} \int_{a}^{b} \frac{e^{x}}{x^{2}} d x \leq \frac{e^{a}+e^{b}}{2}
$$

whose proof is a direct consequence of inequality of Theorem 2.1 and we have

$$
\left(\frac{a+b}{2}\right) \cdot e^{\frac{a+b}{2}} \leq \frac{(1-a) e^{a}+(b-1) e^{b}}{b-a} \leq \frac{a e^{a}+b e^{b}}{2}
$$

which is a direct consequence of inequality of Theorem 2 for $f(x)=e^{x}$ which is not only a harmonic convex function, even it is a harmonic $p$-convex function too. Therefore, for $p \geq 1$, we have

$$
e^{\frac{2^{\frac{1}{p}} a b}{\left.{ }^{p}+b^{p}\right]^{\frac{1}{p}}}} \leq \frac{p(a b)^{p}}{b^{p}-a^{p}} \int_{a}^{b} \frac{e^{x}}{x^{p+1}} d x \leq \frac{e^{a}+e^{b}}{2} .
$$

From above discussion, it is easy to conclude that we have a good estimate of $\int_{a}^{b} \frac{e^{x}}{x^{n}} d x \forall n \in \mathbb{N}$

- Since $f(x)=x^{2} e^{x^{2}}$ is non-decreasing convex function on $(0,1)$, so it is harmonic convex function. Therefore, by using inequality of Theorem 2.1 for $a, b \in(0, \infty)$ and $a \neq b$, we have

$$
\left(\frac{2 a b}{a+b}\right)^{2} e^{\left(\frac{2 a b}{a+b}\right)^{2}} \leq \frac{a b}{b-a} \int_{a}^{b} e^{x^{2}} d x \leq \frac{a^{2} e^{a^{2}}+b^{2} e^{b^{2}}}{2}
$$

for all $a, b \in(0, \infty)$.

- Since, $f(x)=\sin (-x)$ is convex and non-decreasing function in $\left(0, \frac{\pi}{2}\right)$, therefore it is harmonic convex $\forall x \in\left(0, \frac{\pi}{2}\right)$. Therefore, by using inequality of Theorem 2.1 for $a, b \in(0, \infty)$, we have

$$
\frac{\sin a+\sin b}{2} \leq \frac{a b}{b-a} \int_{a}^{b} \frac{\sin x}{x^{2}} d x \leq \sin \left(\frac{2 a b}{a+b}\right)
$$

Similarly, we can estimate $\int_{a}^{b} \frac{\sin x}{x^{n}} d x$ and $\int_{a}^{b} \frac{\cos x}{x^{n}} d x$ for all $n \in \mathbb{N}$ such that $a, b \in(0, \infty)$.

\section{Conclusion}

The harmonic convexity of a function is the basis for many inequalities in mathematics as you may see in this research paper. Furthermore, harmonic convexity provide an analytic tool to estimate several known definite integral like $\int_{a}^{b} \frac{e^{x}}{x^{n}} d x, \int_{a}^{b} e^{x^{2}} d x, \int_{a}^{b} \frac{\sin x}{x^{n}} d x$ and $\int_{a}^{b} \frac{\cos x}{x^{n}} d x \forall n \in \mathbb{N}$, where $a, b \in(0, \infty)$. We have discussed several important aspect of harmonic $p$-convex functions and encourage the interested researcher to explore more interesting results for this class of functions. 


\section{Funding}

The authors are grateful to the Basque Government by its support through Grant IT1207/19. This research article is partially supported by Higher Education Commission of Pakistan too.

\section{Acknowledgement}

The authors wish to express their heartfelt thanks to the referees for their constructive comments and helpful suggestions to improve the final version of this paper.

\section{REFERENCES}

[1] D-Y. Hwang, Some inequalities for differentiable convex mapping with application to weighted trapezoidal formula and higher moments of random variables, Appl. Math. Comput. 217 (2011), 9598-9605.

[2] D-Y. Hwang, Some inequalities for differentiable convex mapping with application to weighted midpoint formula and higher moments of random variables, Appl. Math. Comput. 232 (2014), 68-75.

[3] S.S. Dragomir, Hermite-Hadamard's type inequalities for convex funtions of selfadjoint operators in Hilbert spaces, Linear Algebra Appl. 436 (2012), no. 5, 1503-1515.

[4] S.S. Dragomir and C.E.M. Pearce, Selected topics on Hermite-Hadamard type inequalities and applications, RGMIA Monographs, 2000. Available online at https://rgmia.org/monographs/hermite_hadamard.html.

[5] I. A. Baloch and I. Iscan, Some Hermite-Hadamard type integral inequalities for Harmonically (p, (s,m))-convex functions, J. Inequal. Spec. Funct. 8 (2017), 65-84.

[6] I. A. Baloch, I. Iscan and S. S. Dragomir, Fejer's type inequalities for Harmonically $(s, m)$-convex functions, Int. J. Anal. Appl. 12 (2016), 188-197.

[7] I. A. Baloch, I.Işcan, Some Ostrowski Type Inequalities For Harmonically $(s, m)$-convex functoins in Second Sense, Int. J. Anal. 2015 (2015), Article ID 672675, 9 pages.

[8] Z. B. Fang and R. Shi, On the(p,h)-convex function and some integral inequalities, J. Inequal. Appl., 2014 (2014), Art. ID 45.

[9] İ. İşcan, Hermite-Hadamard type inequaities for harmonically functions, Hacettepe J. Math. Stat. 43 (6) (2014), 935-942.

[10] İ.İşcan, Hermite-Hamard type inequalities for harmonically $(\alpha, m)$-convex functions. arXiv:1307.5402 [math.CA], 2015.

[11] İ. İşcan, Hermite-Hadamard type inequaities for p-convex functions, Int. J. Anal. Appl. 11 (2016), 137-145.

[12] C. P. Niculescu and L. E. Persson (2006). Convex functions and their applications, Springer-Verlag, New York.

[13] K.S. Zhang and J.P. Wan, p-convex functions and their properties, Pure Appl. Math. 23(1) (2007), 130-133.

[14] M. E. Gordji, M. R. Delavar and M. De La Sen, On $\phi$-convex functions, J. Math. Inequal. 10 (2016), 173-183. 